\title{
The effects of an imperfect vaccine on cholera control
}

\author{
Jing'an Cui, Zhanmin Wu, Guohua Song
}

School of Science, Beijing University of Civil Engineering and Architecture, Beijing, China

Email: cuijingan@bucea.edu.cn, zhanminwu@126.com

Received 5 January 2013; revised 10 February 2013; accepted 20 February 2013

\begin{abstract}
In this paper, we consider a SVIR-B cholera model with imperfect vaccination. By analyzing the corresponding characteristic equations, the local asymptotically stability of a disease-free equilibrium and an endemic equilibrium is established. We calculate the certain threshold known as the basic reproduction number $\boldsymbol{R}_{v}$. If $\boldsymbol{R}_{v}<1$, we obtain sufficient conditions for the global asymptotically stability of the diseasefree equilibrium, the diseases will be eliminated from the community. By comparison arguments, it is proved that if $\boldsymbol{R}_{v}>1$, the unique endemic equilibrium is local asymptotically stable. We perform sensitivity analysis of $\boldsymbol{R}_{v}$ on the parameters in order to determine their relative importance to disease control and show that an imperfect vaccine is always beneficial in reducing disease spread within the community.
\end{abstract}

Keywords: Cholera Model; Stability; The Basic Reproduction Number; Sensitivity Analysis

\section{INTRODUCTION}

Cholera is an acute intestinal infection caused by ingestion of food or water contaminated with the bacterium vibrio cholera. Since Koch found vibrio cholera in 1883, the research for cholera vaccine has more than one hundred years. People have developed a variety of vaccines. However, these vaccines were parenteral, which have short effective protection and big side effects. In 1973, the World Health Organization canceled the vaccine inoculation which attracted a major concern to oral vaccines. At present, there are three kinds of oral vaccine (i.e., $\mathrm{WC} / \mathrm{BS}$ vaccine, $\mathrm{WC} / \mathrm{rBS}$ vaccine and $\mathrm{CVD}_{103}-\mathrm{HgR}$ vaccine) have been proved to be safe, effective and immunogenic, which were approved to apply in some countries [1].

In this paper, according to the natural history of cholera, we improve the model of [2] in the following two aspects. Firstly, if the cholera persists for a long time, it will cause the death [3], especially in the area where water and sanitation resources are not adequate [4], a pa- rameter $\mathrm{d}$ is added to describe the rate of disease-related death. Secondly, we propose a proportion of the vaccination in susceptible individuals. As is shown in the following differential equations:

$$
\left\{\begin{aligned}
\frac{\mathrm{d} S(t)}{\mathrm{d} t}= & \mu_{1}-\beta_{1} S(t) B(t)-\beta_{2} S(t) I(t)-\phi S(t) \\
& -\mu_{1} S(t)+\theta V(t), \\
\frac{\mathrm{d} V}{\mathrm{~d} t}= & \phi S(t)-\theta V(t)-\mu_{1} V(t), \\
\frac{\mathrm{d} I(t)}{\mathrm{d} t}= & \beta_{1} S(t) B(t)+\beta_{2} S(t) I(t)-\left(d+\alpha+\mu_{1}\right) I(t) \\
\frac{\mathrm{d} R}{\mathrm{~d} t}=\alpha I(t)-\mu_{1} R(t), & \frac{\mathrm{d} B(t)}{\mathrm{d} t}=\eta I(t)-\mu_{2} B(t) .
\end{aligned}\right.
$$

The flow diagram of the model is depicted in Figure 1. Since the first three and last equations in (1.1) are independent of the variable $R$, it suffices to consider the following reduced model:

$$
\left\{\begin{aligned}
\frac{\mathrm{d} S(t)}{\mathrm{d} t}= & \mu_{1}-\beta_{1} S(t) B(t)-\beta_{2} S(t) I(t)-\phi S(t) \\
& -\mu_{1} S(t)+\theta V(t), \\
\frac{\mathrm{d} V}{\mathrm{~d} t}= & \phi S(t)-\theta V(t)-\mu_{1} V(t), \\
\frac{\mathrm{d} I(t)}{\mathrm{d} t}= & \beta_{1} S(t) B(t)+\beta_{2} S(t) I(t)-\left(d+\alpha+\mu_{1}\right) I(t) \\
\frac{\mathrm{d} B(t)}{\mathrm{d} t}= & \eta I(t)-\mu_{2} B(t) .
\end{aligned}\right.
$$

Here, $S, V, I$ and $R$ refer to the susceptible individuals, vaccinated individuals, infected individuals, and recovered individuals, respectively.

The pathogen population at time $t$, is given by $B(t)$. The parameter $\mu_{1}$ denotes the natural human birth and 


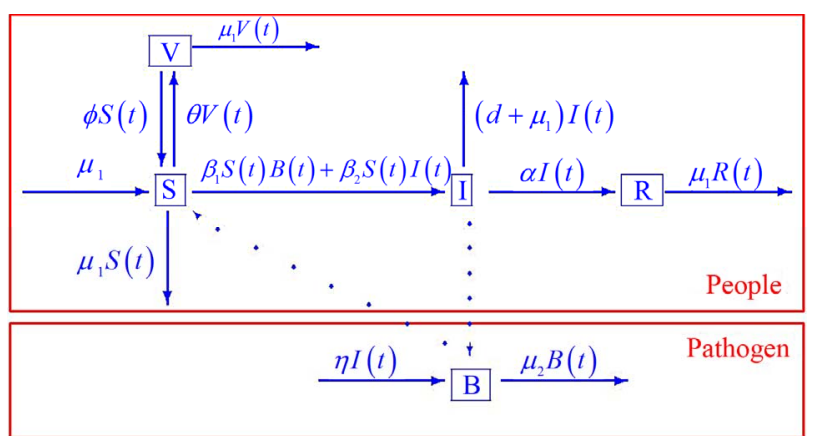

Figure 1. Progression of infection from susceptible $(S)$ and vaccinated $(V)$ individuals through the infected $(I)$ and recovered $(R)$ compartments for the combined human-environment epidemiological model with an environmental component.

death rate, $\alpha$ denotes the rate of recovery from the disease, $\eta$ represents the rate of human contribution to the growth of the pathogen, and $\mu_{2}$ represents the death rate of the pathogen in the environment. The coefficients $\beta_{1}$ and $\beta_{2}$ represent the contact rates for the human-environment and human-human interactions, respectively. The rate at which the susceptible population is vaccinated is $\phi$, and the rate at which the vaccine wears off is $\theta$.

All parameters are assumed non-negative, and the initial conditions of the system (1.2) are assumed as following

$$
S(0)>0, V(0)>0, I(0)>0, B(0)>0 .
$$

The organization of this paper is as follows: the positivity and boundedness of solutions are obtained in Section 2. In Section 3, we firstly calculate the basic reproduction number and obtain the existence of the endemic equilibrium. We get the local and global asymptotically stability of the disease-free equilibrium in Section 4. In Section 5, we show that the local asymptotically stability of the endemic equilibrium. We analyze the sensitivity of $R_{v}$ on the parameters, and we present the numerical simulation in Section 6. The paper ends with a conclusion in Section 7.

\section{POSITIVITY AND BOUNDEDNESS OF SOLUTIONS}

In the following, we show that the solutions of the system (1.2) are positive with the non-negative initial conditions (1.3).

Theorem 2.1. The solutions $(S(t), V(t), I(t), B(t))$ of the model (1.2) are non-negative for all $t>0$ with initial conditions (1.3).

Proof. The system (1.2) can be put into the matrix form

$$
X^{\prime}=M(X),
$$

where $X=(S, V, I, B)^{\mathrm{T}} \in R^{4}$ and $M(X)$ is given by

$$
\begin{aligned}
M(X) & =\left(\begin{array}{l}
M_{1}(X) \\
M_{2}(X) \\
M_{3}(X) \\
M_{4}(X)
\end{array}\right) \\
& =\left(\begin{array}{c}
\mu_{1}-\beta_{1} S(t) B(t)-\beta_{2} S(t) I(t)-\phi S(t) \\
-\mu_{1} S(t)+\theta V(t) \\
\phi S(t)-\theta V(t)-\mu_{1} V(t) \\
\beta_{1} S(t) B(t)+\beta_{2} S(t) I(t)-\left(d+\alpha+\mu_{1}\right) I(t) \\
\eta I(t)-\mu_{2} B(t)
\end{array}\right) .
\end{aligned}
$$

We have

$$
\begin{aligned}
& \left.\frac{\mathrm{d} S}{\mathrm{~d} t}\right|_{S=0}=\mu_{1}+\theta V>0,\left.\frac{\mathrm{d} V}{\mathrm{~d} t}\right|_{V=0}=\phi S>0, \\
& \left.\frac{\mathrm{d} I}{\mathrm{~d} t}\right|_{I=0}=\beta_{1} S B \geq 0,\left.\frac{\mathrm{d} B}{\mathrm{~d} t}\right|_{B=0}=\eta I \geq 0 .
\end{aligned}
$$

Therefore,

$$
\left.M_{i}\right|_{X_{i}=0, X_{i} \in C_{+}^{4}} \geq 0, i=1,2,3,4 .
$$

Due to Lemma 2 in [5], any solution of the system (1.2) is such that $X(T) \in R_{+}^{4}$ for all $t \geq 0$. This completes the proof of Theorem 2.1.

Theorem 2.2. All solutions $(S(t), V(t), I(t), B(t))$ of the model (1.2) are bounded.

Proof. The system (1.2) is split into two parts, the human population (i.e., $S(t), V(t)$, and $I(t))$ and pathogen population (i.e., $B(t))$. It follows from the first three equations of the system (1.2) that

$$
\begin{aligned}
\frac{\mathrm{d}(S+V+I)}{\mathrm{d} t}= & \mu_{1}(1-S-V-I)-\mathrm{d} I-\alpha I \\
& \leq \mu_{1}(1-S-V-I),
\end{aligned}
$$

then it follows that $\limsup _{t \rightarrow+\infty}(S+V+I) \leq 1$. From the first equation, we can get

$$
\frac{\mathrm{d} S}{\mathrm{~d} t} \leq \mu_{1}-\mu_{1} S-\phi S+\theta V \leq \mu_{1}+\theta-\left(\mu_{1}+\theta+\phi\right) S .
$$

Thus $\frac{\mathrm{d} S}{\mathrm{~d} t} \leq 0$, as $S(t) \geq \frac{\mu_{1}+\theta}{\mu_{1}+\theta+\phi}$. It is easy obtain

$$
\frac{\mathrm{d} V}{\mathrm{~d} t} \leq \phi(1-V)-\left(\mu_{1}+\theta\right) V=\phi-\left(\mu_{1}+\theta+\phi\right) V .
$$

$\frac{\mathrm{d} V}{\mathrm{~d} t} \leq 0$, as $V(t) \geq \frac{\phi}{\mu_{1}+\theta+\phi}$. From the last equation, we can obtain

$$
\frac{\mathrm{d} B}{\mathrm{~d} t} \leq \eta-\mu_{2} B
$$


Hence, $\frac{\mathrm{d} B}{\mathrm{~d} t} \leq 0$, when $B \geq \frac{\eta}{\mu_{2}}$. Therefore, all solutions $(S(t), V(t), I(t), B(t))$ of the model (1.2) are bounded.

From above discussion, we can see that the feasible region of human population for system (1.2) is

$$
\begin{aligned}
\Omega_{H}= & \left\{(S, V, I) \mid S+V+I \leq 1,0 \leq S \leq \frac{\mu_{1}+\theta}{\mu_{1}+\theta+\phi},\right. \\
& \left.0 \leq V \leq \frac{\phi}{\mu_{1}+\theta+\phi}, I \geq 0\right\}
\end{aligned}
$$

and the feasible region of pathogen population for system (1.2) is

$$
\Omega_{H}=\left\{B \mid 0 \leq B \leq \frac{\eta}{\mu_{2}}\right\} .
$$

Define $\Omega=\Omega_{H} \times \Omega_{B}$. Let int $\Omega$ denote the interior of $\Omega$. It is easy to verify that the region $\Omega$ is a positively invariant region (i.e., the solutions with initial conditions in $\Omega$ remain in $\Omega$ ) with respect to the system (1.2). Hence, we will consider the global asymptotically stability of (1.2) in region $\Omega$.

\section{THE EXISTENCE OF EQUILIBRIA}

In this section, we investigate the existence of equilibria of system (1.2). Solving the right hand side of the model system (1.2) by equating it to zero, we obtain the following biologically relevant equilibria.

It is easy to see that model (1.2) always has a disease-free equilibrium (the absence of infection, that is, $I=B=0, E_{0}\left(S_{0}, V_{0}, 0,0\right)$, where $S_{0}=\frac{\mu_{1}+\theta}{\mu_{1}+\theta+\phi}$ and $V_{0}=\frac{\phi}{\mu_{1}+\theta+\phi}$. Let $X=(I, B, S, V)^{\mathrm{T}}$. Then model (1.2) can be written as

$$
\frac{\mathrm{d} x}{\mathrm{~d} t}=\mathscr{F}(x)-\mathscr{V}(x),
$$

where

$$
\begin{aligned}
& \mathscr{T}(x)=\left(\begin{array}{c}
\beta_{1} S B+\beta_{2} S I \\
0 \\
0 \\
0
\end{array}\right) \\
& \mathscr{V}(x)=\left(\begin{array}{c}
\left(\mathrm{d}+\alpha+\mu_{1}\right) I \\
-\eta I(t)+\mu_{2} B \\
-\mu_{1}+\beta_{1} S B+\beta_{2} S I+\phi S+\mu_{1} S-\theta V \\
-\phi S+\theta V+\mu_{1} V
\end{array}\right) .
\end{aligned}
$$

We can get

$$
F=\left(\begin{array}{cc}
\beta_{2} S_{0} & \beta_{1} S_{0} \\
0 & 0
\end{array}\right), V=\left(\begin{array}{cc}
\mathrm{d}+\alpha+\mu_{1} & 0 \\
-\eta & \mu_{2}
\end{array}\right),
$$

giving

$$
V^{-1}=\left(\begin{array}{cc}
\frac{1}{\mathrm{~d}+\alpha+\mu_{1}} & 0 \\
\frac{\eta}{\mu_{2}\left(\mathrm{~d}+\alpha+\mu_{1}\right)} & \frac{1}{\mu_{2}}
\end{array}\right) .
$$

$F V^{-1}$ is the next generation matrix for model (1.2). It then follows that the spectral radius of matrix $F V^{-1}$ is $\rho\left(F V^{-1}\right)=\frac{S_{0}\left(\beta_{2} \mu_{2}+\beta_{1} \eta\right)}{\mu_{2}\left(\mathrm{~d}+\alpha+\mu_{1}\right)}$. According to Theorem 2 in [6], the basic reproduction number of model (1.2) is

$$
R_{v}=\frac{\left(\mu_{1}+\theta\right)\left(\beta_{2} \mu_{2}+\beta_{1} \eta\right)}{\mu_{2}\left(\mu_{1}+\theta+\phi\right)\left(\mathrm{d}+\alpha+\mu_{1}\right)} .
$$

In the following, we will discuss the case with $R_{v}>1$. The existence and uniqueness of the endemic equilibrium is established as follows.

The endemic equilibrium $E^{*}\left(S^{*}, V^{*}, I^{*}, B^{*}\right)$ can be deduced by the following system:

$$
\left\{\begin{array}{l}
\mu_{1}-\beta_{1} S^{*} B^{*}-\beta_{2} S^{*} I^{*}-\phi S^{*}-\mu_{1} S^{*}+\theta V^{*}=0 \\
\phi S^{*}-\theta V^{*}-\mu_{1} V^{*}=0 \\
\beta_{1} S^{*} B^{*}+\beta_{2} S^{*} I^{*}-\left(\mathrm{d}+\alpha+\mu_{1}\right) I^{*}=0 \\
\eta I^{*}-\mu_{2} B^{*}=0
\end{array}\right.
$$

which gives,

$$
\begin{aligned}
S^{*} & =\frac{\mu_{1}+\theta}{\mu_{1}+\theta+\phi} \frac{1}{R_{v}}, \\
V^{*} & =\frac{\phi}{\mu_{1}+\theta+\phi} \frac{1}{R_{v}}, \\
I^{*} & =\frac{\mu_{1}}{\mathrm{~d}+\alpha+\mu_{1}}\left(1-\frac{1}{R_{v}}\right), \\
B^{*} & =\frac{\eta \mu_{1}}{\mu_{2}\left(\mathrm{~d}+\alpha+\mu_{1}\right)}\left(1-\frac{1}{R_{v}}\right) .
\end{aligned}
$$

Obviously, when $R_{v}>1, I^{*}>0, B^{*}>0$.

Theorem 3.1. The system (1.2) has a unique endemic equilibrium when $R_{v}>1$ and no positive endemic equilibrium when $R_{v}<1$.

\section{STABILITY OF DISEASE-FREE EQUILIBRIUM}

Now, we will discuss the local and global asymptotically stability of the disease-free equilibrium. From above and [6], we can obtain the following theorem.

Theorem 4.1. The disease-free equilibrium $E_{0}$ is locally asymptotically stable for $R_{v}<1$ and unstable for $R_{v}$ 
$>1$.

Proof. The Jacobian matrix of the system (1.2) at $X=$ $E_{0}$ is

$$
\begin{aligned}
& J\left(E_{0}\right) \\
= & \left(\begin{array}{cccc}
-\mu_{1}-\phi & \theta & -\beta_{2} S_{0} & -\beta_{1} S_{0} \\
\phi & -\theta-\mu_{1} & 0 & 0 \\
0 & 0 & \beta_{2} S_{0}-\left(\mathrm{d}+\alpha+\mu_{1}\right) & \beta_{1} S_{0} \\
0 & 0 & \eta & -\mu_{2}
\end{array}\right)
\end{aligned}
$$

The characteristic polynomial of the matrix $J\left(E_{0}\right)$ is given by

$$
\operatorname{det}\left(\lambda I-J\left(E_{0}\right)\right)=a_{0} \lambda^{4}+a_{1} \lambda^{3}+a_{2} \lambda^{2}+a_{3} \lambda+a_{4},
$$

where

$$
\begin{aligned}
& a_{0}=1 \\
& \begin{aligned}
a_{1}= & 2 \mu_{1}+\mu_{2}+\theta+\phi+\left(\mathrm{d}+\alpha+\mu_{1}-\beta_{2} S_{0}\right), \\
a_{2}= & \left(\theta+2 \mu_{1}+\phi\right)\left(\mathrm{d}+\alpha+\mu_{1}-\beta_{2} S_{0}\right) \\
& +\left[\mu_{2}\left(\mathrm{~d}+\alpha+\mu_{1}\right)-\left(\beta_{1} S_{0} \eta+\beta_{2} S_{0} \mu_{2}\right)\right] \\
& +\mu_{1}^{2}+2 \mu_{1} \mu_{2}+\theta \mu_{2}+\phi \mu_{2}+\phi \mu_{1}+\theta \mu_{1} \\
a_{3}=\left(2 \mu_{1}+\phi+\theta\right) & \times\left[\mu_{2}\left(\mathrm{~d}+\alpha+\mu_{1}\right)-\left(\beta_{1} S_{0} \eta+\beta_{2} \cdot S_{0} \mu_{2}\right)\right] \\
& +\left(\mu_{1} \theta+\mu_{1}^{2}+\phi \mu_{1}\right)\left(d+\alpha+\mu_{1}-\beta_{2} \cdot S_{0}\right) \\
& +\mu_{1}^{2} \mu_{2}+(\theta+\phi) \mu_{1} \mu_{2} \\
a_{4}=\mu_{1} \mu_{2} & \left(\theta+\phi+\mu_{1}\right)\left(\mathrm{d}+\alpha+\mu_{1}\right)\left(1-R_{v}\right), \\
a_{1} a_{2}-a_{3}= & \left(\mathrm{d}+\alpha+\mu_{1}-\beta_{2} S_{0}\right) \\
& \times\left(4 \mu_{1} \theta+4 \phi \mu_{1}+4 \cdot \mu_{1}^{2}+4 \mu_{1} \mu_{2}+\phi^{2}+\theta^{2}\right. \\
& \left.+2 \mu_{2} \phi+2 \mu_{2} \theta+2 \theta \phi\right) \\
& +\left[\mu_{2}\left(\mathrm{~d}+\alpha+\mu_{1}\right)-\left(\beta_{1} S_{0} \eta+\beta_{2} S_{0} \mu_{2}\right)\right] \\
& \times \mu_{2}\left(\mathrm{~d}+\alpha+\mu_{1}-\beta_{2} S_{0}\right)^{2}\left(\theta+\phi+2 \mu_{1}\right) \\
& +4\left(\theta+\phi+\mu_{1}\right) \mu_{1} \mu_{2}+2 \mu_{1} \mu_{2}^{2}+\theta \mu_{2}^{2} \\
& +3(\phi+\theta) \mu_{1}^{2}+\left(\phi^{2}+\theta^{2}\right) \mu_{2}+\theta^{2} \mu_{1}^{2} \\
& +2 \theta \phi\left(\mu_{2}+\mu_{1}\right)+2 \mu_{1}^{3} .
\end{aligned}
\end{aligned}
$$

If $R_{v}<1$, then

$$
\mu_{2}\left(\mathrm{~d}+\alpha+\mu_{1}\right)>\beta_{2} S_{0} \mu_{2}+\beta_{1} S_{0} \eta,
$$

further

$$
\mathrm{d}+\alpha+\mu_{1}>\beta_{2} S_{0}
$$

After some calculations, if $R_{v}<1$ we have $a_{1}>0, a_{2}>$ $0, a_{3}>0, a_{4}>0, a_{1} a_{2}-a_{3}>0, a_{1} a_{2} a_{3}>a_{3}^{2}+a_{1}^{2} a_{4}$ (see Appendix A). Thus, using the Routh-Hurwitz criterion, all eigenvalues of $J\left(E_{0}\right)$ have negative real part, $E_{0}$ is local asymptotically stable for the system (1.2). If $R_{v} \geq 1$, then $a_{4} \leq 0$ and we show that $J\left(E_{0}\right)$ has at least one eigenvalues with non-negative real part. Consequently, $E_{0}$ is not stable.

Theorem 4.2. When $R_{v}<1$ the disease-free equilibrium is globally asymptotically stable.

We will prove the global asymptotically stability of the disease-free equilibrium using Lemma 4.1.

Lemma 4.1. [7] If a model system can be written in the form

$$
\left\{\begin{array}{l}
\frac{\mathrm{d} \mathcal{X}}{\mathrm{d} t}=P(\mathcal{X}, \mathcal{Z}), \\
\frac{\mathrm{d} \mathcal{Z}}{\mathrm{d} t}=G(\mathcal{X}, \mathcal{Z}), G(\mathcal{Z}, 0)=0,
\end{array}\right.
$$

where $\mathcal{X} \in R^{m}$ denotes(its components) the number of uninfected individuals and $\mathcal{Z} \in R^{n}$ denotes (its components) the number of infected individuals including latent, etc. $U_{0}=\left(x^{*}, 0\right)$ denotes the disease-free equilibrium of the system.

And assume that

(H1) $\frac{\mathrm{d} X}{\mathrm{~d} t}=P(X, 0), \quad X^{*}$ is globally asymptotically stable;

(H2) $G(X, Z)=A Z-\hat{G}(X, Z), \hat{G}(X, Z) \geq 0$, for

$(X, \mathcal{Z}) \in \Omega$, where the Jacobian matrix $A=\frac{\partial G}{\partial \mathcal{Z}}\left(\boldsymbol{X}^{*}, 0\right)$ is an Metzler matrix(the off-diagonal elements of $A$ are non-negative) and $\Omega$ is the region where the model makes biological sense. Then the fixed point $U_{0}=\left(X^{*}, 0\right)$ is a globally asymptotically stable equilibrium of cholera model system (1.2) provided that $R_{v}<1$.

We begin by showing condition (H1) as

$$
P(x, 0)=\left(\begin{array}{c}
\mu_{1}-\mu_{1} S-\phi \mu_{1}+\theta V \\
\phi S-\theta V-\mu_{1} V
\end{array}\right) .
$$

For the equilibrium $U_{0}=\left(x^{*}, 0\right)$, the system reduces to

$$
\left\{\begin{array}{l}
\frac{\mathrm{d} S(t)}{\mathrm{d} t}=\mu_{1}-\phi S(t)-\mu_{1} S(t)+\theta V(t), \\
\frac{\mathrm{d} V}{\mathrm{~d} t}=\phi S(t)-\theta V(t)-\mu_{1} V(t) .
\end{array}\right.
$$

The characteristic polynomial of the system is given by

$$
\left(\lambda+\mu_{1}\right)\left(\lambda+\theta+\phi+\mu_{1}\right)=0 .
$$

There are two negative characteristic foots are $\lambda=-\mu_{1}$, $\lambda=-\theta-\phi-\mu_{1}$. Hence, $X^{*}$ is always globally asymptotically stable.

Next, applying Lemma 4.1 to the cholera model system (1.2) gives 


$$
\begin{aligned}
G(\mathcal{X}, \mathcal{Z}) & =A \mathcal{Z}-G(\mathcal{X}, \mathcal{Z}) \\
& =\left(\begin{array}{cc}
\beta_{2} S_{0}-\left(\mathrm{d}+\alpha+\mu_{1}\right) & \beta_{1} S_{0} \\
\eta & -\mu_{2}
\end{array}\right)\left(\begin{array}{l}
I \\
B
\end{array}\right)-\left(\begin{array}{l}
0 \\
0
\end{array}\right),
\end{aligned}
$$

which is clearly an Metzler matrix. Meanwhile, we find $\hat{G}(\mathcal{X}, \mathcal{Z})=0$. Hence, the disease-free equilibrium is globally asymptotically stable.

\section{STABILITY OF THE ENDEMIC EQUILIBRIUM}

Now we consider the case with $R_{v}>1$. The stability of the endemic equilibrium is established as follows:

Theorem 5.1. If $R_{v}>1, E^{*}\left(S^{*}, V^{*}, I^{*}, B^{*}\right)$ is locally asymptotically stable.

Proof. Let

$$
J_{1}=\beta_{1} B^{*}+\beta_{2} I^{*}, \quad J_{2}=\beta_{2} S^{*}, \quad J_{3}=\beta_{1} S^{*} .
$$

The Jacobian matrix at $E^{*}\left(S^{*}, V^{*}, I^{*}, B^{*}\right)$ is

$$
\begin{aligned}
& J\left(E^{*}\right) \\
= & \left(\begin{array}{cccc}
-J_{1}-\mu_{1}-\phi & \theta & -J_{2} & -J_{3} \\
\phi & -\theta-\mu_{1} & 0 & 0 \\
0 & 0 & J_{2}-\left(\mathrm{d}+\alpha+\mu_{1}\right) & J_{3} \\
0 & 0 & \eta & -\mu_{2}
\end{array}\right)
\end{aligned}
$$

The characteristic polynomial of the matrix $J\left(E^{*}\right)$ is given by

$$
\operatorname{det}\left(\lambda I-J\left(E^{*}\right)\right)=b_{0} \lambda^{4}+b_{1} \lambda^{3}+b_{2} \lambda^{2}+b_{3} \lambda+b_{4},
$$

where

$$
\begin{aligned}
b_{0}= & 1, \\
b_{1}= & J_{1}+2 \mu_{1}+\mu_{2}+\theta+\phi+\left(\mathrm{d}+\alpha+\mu_{1}-J_{2}\right), \\
b_{2}= & \left(\mathrm{d}+\alpha+\mu_{1}-J_{2}\right)\left(\theta+2 \mu_{1}+\phi\right) \\
& +\left[\mu_{2}\left(\mathrm{~d}+\alpha+\mu_{1}\right)-\left(J_{2} \mu_{2}+J_{3} \eta\right)\right] \\
& +J_{3} \mu_{1}^{2}+2 \mu_{1} \mu_{2}+\theta \mu_{2}+\phi \mu_{1}+\phi \mu_{2} \\
& +\theta \mu_{1}+\alpha J_{1}+2 \mu_{1} J_{1}+\mu_{2} J_{2}+d J_{1}, \\
b_{3}= & \left(\mathrm{d}+\alpha+\mu_{1}-J_{2}\right)\left(\mu_{1} \theta+\mu_{1}^{2}+\mu_{1} \phi\right) \\
& +\left[\mu_{2}\left(\mathrm{~d}+\alpha+\mu_{1}\right)-\left(J_{2} \mu_{2}+J_{3} \eta\right)\right]\left(2 \mu_{1}+\phi+\theta\right) \\
& +J_{3} \cdot \mu_{1}^{2}+\mu_{1}^{2} \mu_{2}+\theta \mu_{1} \mu_{2}+\phi \mu_{1} \mu_{2}+\theta \alpha J_{1}+\alpha \mu_{1} J_{1} \\
& +J_{1} \mu_{1}^{2}+\mathrm{d} \mu_{1} J_{1}+2 \mu_{1} \mu_{2} J_{1}+(\mathrm{d}+\alpha+\theta) \mu_{2} \cdot J_{1} \\
& +\mathrm{d} \theta J_{1}+\theta \mu_{1} J_{1}, \\
b_{4}= & {\left[\mu_{2}\left(\mathrm{~d}+\alpha+\mu_{1}\right)-\left(J_{2} \mu_{2}+J_{3} \eta\right)\right]\left(\phi+\mu_{1}+\theta\right) \cdot \mu_{1} } \\
& +\theta \alpha \mu_{2} J_{1}+\left(\mathrm{d}+\alpha+\mu_{1}+\theta\right) \mu_{1} \mu_{2} J_{1}+\mathrm{d} \theta \cdot \mu_{2} J_{1},
\end{aligned}
$$

$$
\begin{aligned}
b_{1} b_{2}-b_{3} \\
=\left(\mathrm{d}+\alpha+\mu_{1}-J_{2}\right) \\
\quad \times\left(4 \mu_{1} \theta+4 \mu_{1}^{2}+4 \mu_{1} \phi+\theta^{2}+\phi^{2}+2 \mu_{2} \phi+4 \mu_{1} \mu_{2}+\alpha J_{1}\right. \\
\left.+2 \theta \mu_{2}+\theta J_{1}+\phi J_{1}+2 \theta \phi+4 \mu_{1} J_{1}+\mu_{2} J_{1}+\mathrm{d} J_{1}\right) \\
+\left[\mu_{2}\left(\mathrm{~d}+\alpha+\mu_{1}\right)-\left(J_{2} \mu_{2}+J_{3 \eta}\right)\right]\left(\mu_{2}+J_{1}\right) \\
+\left(\mathrm{d}+\alpha+\mu_{1}-J_{2}\right)^{2}\left(\theta+\phi+2 \mu_{1}\right)+(\mathrm{d}+\alpha) \mu_{1} J_{1} \\
+\theta \mu_{2} J_{1}+4\left(\theta+\phi+J_{1}\right) \mu_{1} \mu_{2} \phi+\left(\alpha+2 \mu_{1}+\mu_{2}+\mathrm{d}\right) J_{1}^{2} \\
+3(\phi+\theta) \mu_{1}^{2}+\phi^{2}\left(\mu_{1}+\mu_{2}\right)+\theta^{2}\left(\mu_{1}+\mu_{2}\right)+3 \phi \mu_{1} J_{1} \\
+2 \theta \phi \mu_{2}+\alpha \phi J_{1}+2 \phi \mu_{2} J_{1}+\mathrm{d} \phi J_{1}+2 \theta \cdot \phi \mu_{1}+2 \mu_{1}^{3} .
\end{aligned}
$$

Based on Eq.3.3 and Eq.3.4, we have $\mu_{2}\left(\mathrm{~d}+\alpha+\mu_{1}\right)=\mu_{2} \beta_{2} S^{*}+\eta \beta_{1} S^{*}$. It is then easy to observe that

$$
\mu_{2}\left(\mathrm{~d}+\alpha+\mu_{1}\right)=\mu_{2} J_{2}+\eta J_{3},
$$

further,

$$
\mathrm{d}+\alpha+\mu_{1}>J_{2} .
$$

After some calculations, we have $b_{1}>0, b_{2}>0$,

$$
b_{3}>0, b_{4}>0, b_{1} b_{2}-b_{3}>0, b_{1} b_{2} b_{3}>b_{3}^{2}+b_{1}^{2} b_{4},
$$

(see Appendix B). Using the well-known Routh-Hurwitz criterion, the proof is thus complete.

\section{SENSITIVITY ANALYSIS OF $\boldsymbol{R}_{v}$}

To facilitate the interpretation of the sensitivity of $R_{v}$, we now present some numerical simulations by using the set of parameters values in Table 1.

Now, we regard the vaccinated rate $\phi$ and the wanning rate $\theta$ as the control parameter, while the other parameters are fixed. From Figures 2 and 3, the effects of various parameters, i.e., $\phi$ and $\theta$ on the basic reproduction number $R_{v}$ have been shown. It is noted that as the parameter $\phi$ increases, $R_{v}$ decreases; as $\theta$ decreases, $R_{v}$ decreases. In fact, we can obtain the critical values of $\phi$ and $\theta$ that reduce $R_{v}$ to 1 ,

$$
\begin{aligned}
\phi= & -\frac{-\mu_{1} \mu_{2} \beta_{2}-\mu_{1} \beta_{1} \eta-\theta \mu_{2} \beta_{2}-\theta \eta \beta_{1}}{\mu_{2}\left(\mathrm{~d}+\alpha+\mu_{1}\right)} \\
& +\frac{\left(\mathrm{d}+\alpha+\theta+\mu_{1}\right) \mu_{1} \mu_{2}+d \theta \mu_{2}+\alpha \theta \mu_{2}}{\mu_{2}\left(\mathrm{~d}+\alpha+\mu_{1}\right)},
\end{aligned}
$$

and

$$
\begin{aligned}
\theta= & -\frac{-\mu_{1} \mu_{2} \beta_{2}-\mu_{1} \beta_{1} \eta+}{-\mu_{2} \beta_{2}-\beta_{1} \eta+\mu_{2}\left(\mathrm{~d}+\alpha+\mu_{1}\right)} \\
& +\frac{\left(\mathrm{d}+\alpha+\phi+\mu_{1}\right) \mu_{1} \mu_{2}+\mathrm{d} \phi \mu_{2}+\alpha \phi \mu_{2}}{-\mu_{2} \beta_{2}-\beta_{1} \eta+\mu_{2}\left(\mathrm{~d}+\alpha+\mu_{1}\right)},
\end{aligned}
$$


Table 1. Estimation of parameters.

\begin{tabular}{|c|c|c|c|}
\hline Parameters & Meaning & Values & Reference \\
\hline$\mu_{1}$ & Natural human birth and death rate & $9.13 \times 10^{-5} /$ day & [8] \\
\hline$\beta_{1}$ & Contact rates for the human-environment interaction & $0.214 /$ day & {$[8]$} \\
\hline$\beta_{2}$ & Contact rates for the human-human interaction & $0.02 /$ day & {$[8]$} \\
\hline d & Disease-induced death rate & $0.013 /$ day & [4] \\
\hline$\alpha$ & Recovery Rate at which people recover from environment & $0.2 /$ day & [8] \\
\hline$\eta$ & Contribution of infected individuals to the population of vibrio cholera & 10 & [9] \\
\hline$\mu_{2}$ & Net death rate of vibrio cholera & $0.33 /$ day & [9] \\
\hline
\end{tabular}

In Figure 2, we select $\theta=0.07,0.03,0.007,0.0001$, corresponding $\phi_{v}=2.07,0.89,0.21,0.01$, respectively. We can see that when the wanning rate $\theta$ has a greater value, $\phi_{v}$ has not reasonable value so that when $\phi>\phi_{v}, R_{v}$ $<1$. Similarly, in Figure 3, we select $\phi=0.01,0.1,0.3$, $0.6,0.99$, corresponding $\theta_{v}=0.0002,0.003,0.01,0.02$, 0.03 , respectively. We can see that when $\phi$ is smaller, $\theta_{v}$ has not reasonable value so that when $\theta<\theta_{v}, R_{v}<1$. Thus, the basic reproduction number can not reduces below unity only by increasing $\theta$ or decreasing $\phi$. The critical values $\phi_{v}$ and $\theta_{v}$ play a key role in regulation the infection magnitude. In order to reduce $R_{v}$ to 1 , a greater vaccinated rate than $\phi_{v}$ and a smaller wanning rate than $\theta_{v}$ have to be achieved simultaneously. We will deduce $R_{v}$ below 1 by using both $\phi$ and $\theta$ at the same time, which can control cholera (see Figure 4).

\section{CONCLUSION}

In this paper, we have conducted stability analysis of a SVIR-B cholera model. The mathematical analysis results show that the basic reproduction number $R_{v}$ satisfies a threshold property with threshold value $1 . R_{v}$ in our model include the parameters $\phi$ and $\theta$ which reflect the effect of vaccination. Numerical simulation show also that the vaccination is always beneficial to the eradication of cholera.

However, there are inherent disadvantages towards the vaccination modeling. For cholera with incubation period, it is hard to rapidly identify those with ambiguous symptoms [4]. Moreover, the vaccination does not always work well due to the limitations of medical development level and financial budget (some vaccine is very expensive and some portion of people cannot be covered) [10].

Hence, incorporating some other control strategies, for example, public health improvement, isolation etc, we may consider the more realistic ordinary differential equation model. The theoretical study of cholera models has been in progress, and is an exciting area of future research.

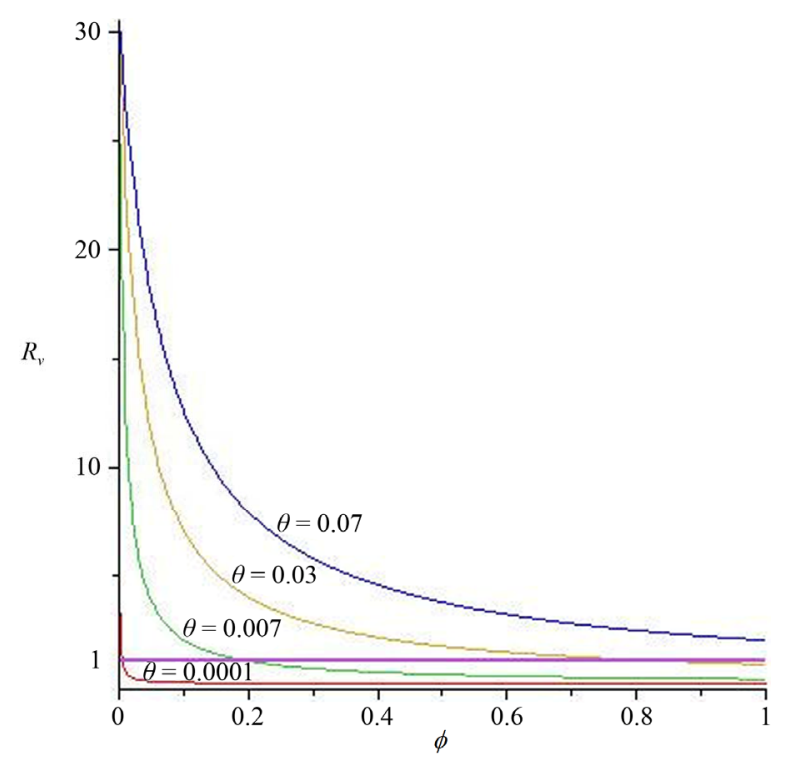

Figure 2. The contour diagram of the basic reproduction number $R_{v}$ with $\phi, \theta$ has some fixed value.

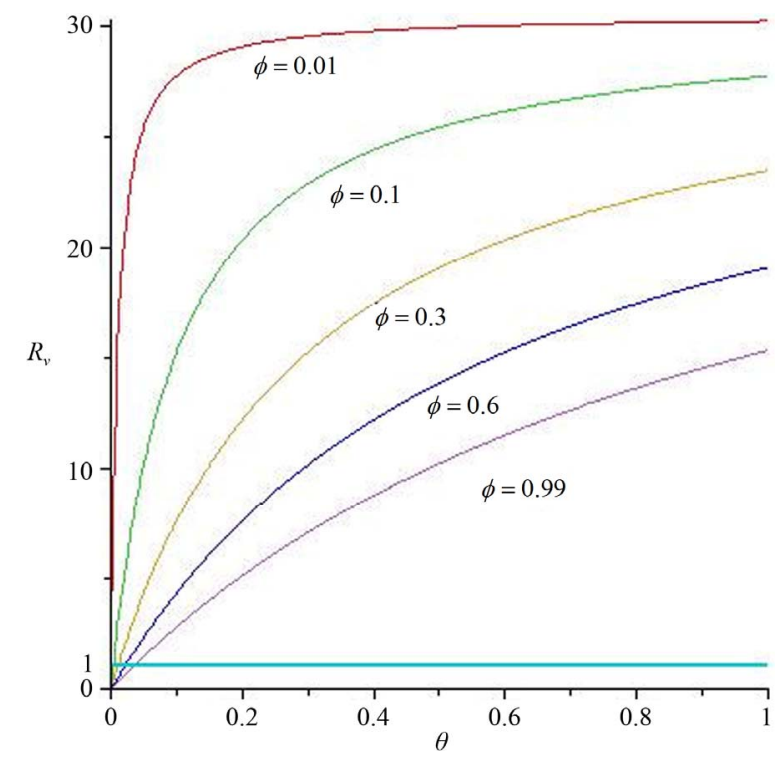

Figure 3. The contour diagram of the basic reproduction number $R_{v}$ with $\phi, \theta$ has some fixed value. 


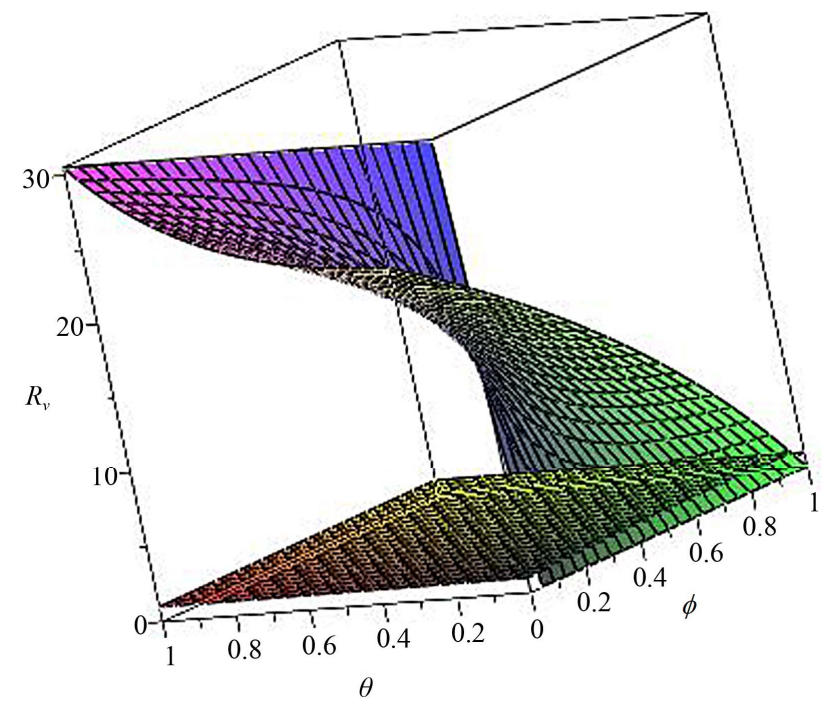

Figure 4. The contour diagram of the basic reproduction number $R_{v}$ with $\phi, \theta$ variables. all the other parameter value are the same as those in Figure 2.

\section{ACKNOWLEDGEMENTS}

This work is supported by the National Natural Science Foundation of China (no.11071011), Funding Project for Academic Human Resources Development in Institutions of Higher Learning Under the Jurisdiction of Beijing Municipality (no.PHR201107123).

\section{REFERENCES}

[1] Kang, Y. and Zhang, H.Z. (2005) The study status of oral cholera vaccine. Chinese Frontier Health Quarantine, 28, 91-92.

[2] Liao, S. and Wang, J. (2007) Global stability analysis of epidemiological models based on Volterra-Lyapunov stable matrices. Chaos, Solitons \& Fractals, 45, 966-977. doi:10.1016/j.chaos.2012.03.009

[3] Singh, S., Chandra, P. and Shukla, J.B. (2003) Modeling and analysis of the spread of carrier dependent infectious diseases with environmental effects. International Journal of Biomathematics, 11, 325-335.

[4] World Health Organization (2012). http://www.who.int/gho/epidemic \_diseases/cholera/en/in dex.html

[5] Yang, X. and Chen, L. (1996) Permanence and positive periodic solution for the single-species nonautonomous delay diffusive models. Computers \& Mathematics with Applications, 32, 109-116. doi:10.1016/0898-1221(96)00129-0

[6] Van den Driessche, P. and Watmough, J. (2002) Reproduction numbers and sub-threshold endemic equilibria for compartmental models of disease transmission. Mathematical Biosciences, 180, 29-48. doi:10.1016/S0025-5564(02)00108-6

[7] Castillo-Chavez, C., Feng, Z.L. and Huang, W.H. (2003) On the computation of $R_{0}$ and its role on global stability. Mathematical Approaches for Emerging and Reemerging Infectious Disease: An introduction, 65, 229-250.

[8] Harley, D.M., Morris, J.G. and Smith, D.L. (2006) Hyperinfectivity: A critical element in the ability of $V$. cholerae to cause epidemics? PLoS Medicine, 3, 63-69.

[9] Codeco, C.T. (2001) Endemic and epidemic dynamics of cholera: The role of the aquatic reservoir. BMC Infectious Diseases, 1, 1. doi:10.1186/1471-2334-1-1

[10] Chen, M. (2011) The opinion of WHO about cholera vaccine. International Journal of Biologicals, 34, 214-218. 


\section{APPENDIX A}

$$
\begin{aligned}
& a_{1} a_{2} a_{3}-a_{3}^{2}-a_{1}^{2} a_{4} \\
& =\left(\mathrm{d}+\alpha+\mu_{1}-\beta_{2} S_{0}\right)^{3}\left(\theta^{2} \mu_{1}+\phi^{2} \mu_{1}+3 \theta \mu_{1}^{2}+2 \mu_{1}^{3}+2 \theta \phi \mu_{1}+3 \phi \mu_{1}^{2}\right) \\
& +\left(\mathrm{d}+\alpha+\mu_{1}-\beta_{2} S_{0}\right)^{2}\left\{\phi^{3} \mu_{1}+4 \theta \mu_{1}\left[\mu_{2}\left(\mathrm{~d}+\alpha+\mu_{1}\right)-\left(\beta_{2} S_{0} \mu_{2}+\beta_{1} S_{0} \eta\right)\right]\right. \\
& +4 \mu_{1}^{2}\left[\mu_{2}\left(\mathrm{~d}+\alpha+\mu_{1}\right)-\left(\beta_{2} S_{0} \mu_{2}+\beta_{1} S_{0} \eta\right)\right]+3 \phi^{2} \mu_{1} \mu_{2}+9 \phi \mu_{1}^{2} \mu_{2}+4 \mu_{1}^{4}+10 \theta \phi \mu_{1}^{2} \\
& +\theta^{2}\left[\mu_{2}\left(\mathrm{~d}+\alpha+\mu_{1}\right)-\left(\beta_{2} S_{0} \mu_{2}+\beta_{1} S_{0} \eta\right)\right]+8 \phi \mu_{1}^{3}+6 \theta \phi \mu_{1} \mu_{2}+5 \phi^{2} \mu_{1}^{2} \\
& +\phi^{2}\left[\mu_{2}\left(\mathrm{~d}+\alpha+\mu_{1}\right)-\left(\beta_{2} S_{0} \mu_{2}+\beta_{1} S_{0} \eta\right)\right]+9 \theta \mu_{1}^{2} \mu_{2}+3 \theta \phi^{2} \mu_{1}+8 \theta \mu_{1}^{3} \\
& +4 \phi \mu_{1}\left[\mu_{2} \cdot\left(\mathrm{d}+\alpha+\mu_{1}\right)-\left(\beta_{2} S_{0} \mu_{2}+\beta_{1} S_{0} \eta\right)\right]+5 \theta^{2} \mu_{1}^{2}+2 \theta \cdot \phi\left[\mu_{2}\left(\mathrm{~d}+\alpha+\mu_{1}\right)-\left(\beta_{2} S_{0} \mu_{2}+\beta_{1} S_{0} \eta\right)\right] \\
& \left.+3 \theta^{2} \phi \mu_{1}+6 \mu_{1}^{3} \mu_{2}+\theta^{3} \mu_{1}+3 \theta^{2} \mu_{1} \mu_{2}\right\}+\left(\mathrm{d}+\alpha+\mu_{1}-\beta_{2} S_{0}\right)\left\{4 \phi^{2} \mu_{1}^{3}+7 \mathrm{~d} \theta \phi \mu_{1}+3 \phi \theta^{2} \mu_{1}^{2}+10 \phi^{2} \mu_{1}^{2} \mu_{2}+2 \theta^{3} \mu_{1} \cdot \mu_{2}\right. \\
& +8 \theta \phi \mu_{1}^{3}+9 \theta \mu_{1}^{2} \mu_{2}^{2}+6 \mu_{1}^{3} \mu_{1}^{2}+16 \phi \mu_{1}^{3} \mu_{2}+4 \cdot \mu_{1}^{3}\left[\mu_{2}\left(\mathrm{~d}+\alpha+\mu_{1}\right)-\left(\beta_{2} S_{0} \mu_{2}+\beta_{1} S_{0} \eta\right)\right]+6 \phi \theta^{2} \mu_{1} \cdot \mu_{2}+2 \mu_{1}^{5} \\
& +8 \phi \mu_{1} \mu_{2}\left[\mu_{2}\left(\mathrm{~d}+\alpha+\mu_{1}\right)-\left(\beta_{2} S_{0} \mu_{2}+\beta_{1} S_{0} \eta\right)\right]+4 \theta^{2} \mu_{1}\left[\mu_{2}\left(\mathrm{~d}+\alpha+\mu_{1}\right)-\left(\beta_{2} S_{0} \mu_{2}+\beta_{1} \cdot S_{0} \eta\right)\right] \\
& +\phi\left[\mu_{2}\left(\mathrm{~d}+\alpha+\mu_{1}\right)-\left(\beta_{2} S_{0} \mu_{2}+\beta_{1} S_{0} \eta\right)\right]^{2}+4 \phi^{2} \mu_{1} \cdot\left[\mu_{2}\left(\mathrm{~d}+\alpha+\mu_{1}\right)-\left(\beta_{2} S_{0} \mu_{2}+\beta_{1} S_{0} \eta\right)\right]+20 \cdot \theta \phi \mu_{1}^{2} \mu_{2} \\
& +8 \theta \mu_{1} \mu_{2}\left[\mu_{2}\left(\mathrm{~d}+\alpha+\mu_{1}\right)-\left(\beta_{2} S_{0} \mu_{2}+\beta_{1} \cdot S_{0} \eta\right)\right]+3 \cdot \theta^{2} \mu_{1} \mu_{2}^{2}+9 \phi \mu_{1}^{2} \mu_{2}^{2}+16 \theta \mu_{1}^{3} \mu_{2}+5 \phi \mu_{1}^{4}+5 \theta \mu_{1}^{4}+2 \phi^{3} \mu_{1} \mu_{2} \\
& +3 \phi^{2} \mu_{1} \mu_{2}^{2}+5 \mathrm{~d} \phi \mu_{1} \mu_{2}+6 \theta \mu_{1}^{2} \cdot\left[\mu_{2}\left(\mathrm{~d}+\alpha+\mu_{1}\right)-\left(\beta_{2} S_{0} \mu_{2}+\beta_{1} S_{0} \eta\right)\right]+2 \phi^{2} \mu_{2}\left[\mu_{2} \cdot\left(\mathrm{d}+\alpha+\mu_{1}\right)-\left(\beta_{2} S_{0} \mu_{2}+\beta_{1} S_{0} \eta\right)\right] \\
& +\phi^{3}\left[\mu_{2}\left(\mathrm{~d}+\alpha+\mu_{1}\right)-\left(\beta_{2} S_{0} \mu_{2}+\beta_{1} S_{0} \eta\right)\right]+\phi \mu_{2}\left[\mu_{2}\left(\mathrm{~d}+\alpha+\mu_{1}\right)-\left(\beta_{2} S_{0} \mu_{2}+\beta_{1} S_{0} \cdot \eta\right)\right] \\
& +2 \theta^{2} \mu_{2}\left[\mu_{2}\left(\mathrm{~d}+\alpha+\mu_{1}\right)-\left(\beta_{2} \cdot S_{0} \mu_{2}+\beta_{1} S_{0} \eta\right)\right]+6 \phi \mu_{1}^{2}\left[\mu_{2}\left(\mathrm{~d}+\alpha+\mu_{1}\right)-\left(\beta_{2} S_{0} \mu_{2}+\beta_{1} S_{0} \eta\right)\right] \\
& +4 \theta \phi \mu_{2} \cdot\left[\mu_{2}\left(\mathrm{~d}+\alpha+\mu_{1}\right)-\left(\beta_{2} S_{0} \mu_{2}+\beta_{1} S_{0} \eta\right)\right]+4 \theta^{2} \mu_{2}^{3}+4 \cdot \theta^{2} \mu_{2}^{2}+\phi^{3} \mu_{1}^{2}+3 \theta \phi^{2} \mu_{1}^{2}+\theta^{3} \mu_{1}^{2}+8 \mu_{1}^{4} \mu_{2} \\
& +2 \mu_{1}\left[\mu_{2}\left(\mathrm{~d}+\alpha+\mu_{1}\right)-\left(\beta_{2} S_{0} \mu_{2}+\beta_{1} S_{0} \eta\right)\right]+3 \theta \phi^{3}\left[\mu_{2}\left(\mathrm{~d}+\alpha+\mu_{1}\right)-\left(\beta_{2} S_{0} \mu_{2}+\beta_{1} S_{0} \eta\right)\right]+10 \theta^{2} \mu_{1}^{2} \mu_{2} \\
& +3 \theta^{2} \phi\left[\mu_{2}\left(\mathrm{~d}+\alpha+\mu_{1}\right)-\left(\beta_{2} S_{0} \mu_{2}+\beta_{1} \cdot S_{0} \eta\right)\right]+8 \theta \phi \mu_{1}\left[\mu_{2}\left(\mathrm{~d}+\alpha+\mu_{1}\right)-\left(\beta_{2} S_{0} \mu_{2}+\beta_{1} S_{0} \eta\right)\right] 6 \theta \phi \mu_{1} \mu_{2}^{2} \\
& +\theta^{3}\left[\mu_{2}\left(\mathrm{~d}+\alpha+\mu_{1}\right)-\left(\beta_{2} S_{0} \mu_{2}+\beta_{1} S_{0} \eta\right)\right]+\theta\left[\mu_{2}\left(\mathrm{~d}+\alpha+\mu_{1}\right)-\left(\beta_{2} S_{0} \mu_{2}+\beta_{1} S_{0} \eta\right)\right] \\
& \left.+8 \mu_{1}^{2} \mu_{2}\left[\mu_{2}\left(\mathrm{~d}+\alpha+\mu_{1}\right)-\left(\beta_{2} S_{0} \mu_{2}+\beta_{1} S_{0} \eta\right)\right]\right\}+\left[\mu_{2}\left(\mathrm{~d}+\alpha+\mu_{1}\right)-\left(\beta_{2} S_{0} \mu_{2}+\beta_{1} S_{0} \eta\right)\right]^{2}\left\{\phi \mu_{2}+2 \mu_{1} \mu_{2}+\theta \mu_{2}\right\} \\
& +\left[\mu_{2}\left(\mathrm{~d}+\alpha+\mu_{1}\right)-\left(\beta_{2} S_{0} \mu_{2}+\beta_{1} S_{0} \eta\right)\right]\left\{8 \theta \phi \mu_{1} \mu_{2}+4 \phi \cdot \mu_{1} \mu_{2}^{2}+\phi^{2} \mu_{2}^{2}+4 \theta^{2} \mu_{1} \mu_{2}+4 \mu_{1}^{3} \mu_{2}+6 \theta \mu_{1}^{2} \mu_{2}+4 \mu_{1}^{2} \cdot \mu_{2}^{2}\right. \\
& \left.+\phi^{3} \mu_{2}+3 \theta \phi^{2} \mu_{2}+2 \theta \phi \mu_{2}^{2}+4 \phi^{2} \mu_{1} \mu_{2}+4 \theta \mu_{1} \mu_{2}^{2}+\theta^{3} \mu_{2}+6 \phi \mu_{1}^{2} \mu_{2}+3 \theta^{2} \phi \mu_{2}+\theta^{2} \mu_{2}^{2}+\theta^{3} \mu_{1} \mu_{2}^{2}+\theta^{3} \cdot \mu_{1}^{2} \mu_{2}\right\} \\
& +3 \theta \phi^{2} \mu_{1} \mu_{2}^{2}+4 \theta^{2} \mu_{1}^{3} \mu_{2}+\phi^{3} \mu_{1}^{2} \mu_{2}+\phi^{3} \mu_{1} \cdot \mu_{2}^{2}+8 \theta \phi \mu_{1}^{3} \mu_{2}+3 \phi \theta^{2} \mu_{1}^{2} \mu_{2}+5 \phi \mu_{1}^{4} \mu_{2}+2 \mu_{1}^{3} \mu_{2}^{3}+5 \theta \mu_{1}^{4} \mu_{2}+4 \mu_{1}^{4} \mu_{2}^{2}+\theta^{2} \mu_{1} \mu_{2}^{3} \\
& +10 \theta \phi \mu_{1}^{2} \mu_{2}^{2}+3 \theta \phi^{2} \cdot \mu_{1}^{2} \mu_{2}+3 \phi \theta^{2} \mu_{1} \mu_{2}^{2}+2 \theta \phi \mu_{1} \mu_{2}^{3}+8 \mu_{1}^{3} \mu_{2}^{2}(\theta+\phi)+5 \mu_{1}^{2} \mu_{2}^{2}\left(\theta^{2}+\phi^{2}\right) \\
& +4 \phi^{2} \mu_{1}^{3} \mu_{2}+3 \mu_{1}^{2} \mu_{2}^{3}(\theta+\phi)+\phi^{2} \mu_{1} \mu_{2}^{3}
\end{aligned}
$$

From Section 4, we know that $R_{v}<1$,

$$
\mu_{2}\left(\mathrm{~d}+\alpha+\mu_{1}\right)>\beta_{2} S_{0} \mu_{2}+\beta_{1} S_{0} \eta
$$

and

$$
\mathrm{d}+\alpha+\mu_{1}>\beta_{2} S_{0} .
$$

After some algebraic manipulations, we have $a_{1} a_{2} a_{3}-a_{3}^{2}-a_{1}^{2} a_{4}>0$, Thus, $a_{1} a_{2} a_{3}>a_{3}^{2}+a_{1}^{2} a_{4}$, when $R_{v}<1$. 


\section{APPENDIX B}

$b_{1} b_{2} b_{3}-b_{3}^{2}-b_{1}^{2} b_{4}$ $=\left(\mathrm{d}+\alpha+\mu_{1}-J_{2}\right)^{3}\left(\theta^{2} \mu_{1}+\phi^{2} \mu_{1}+3 \theta \mu_{1}^{2}+2 \mu_{1}^{3}+2 \theta \phi \mu_{1}+3 \phi \mu_{1}^{2}\right)$

$+\left(\mathrm{d}+\alpha+\mu_{1}-J_{2}\right)^{2}\left\{\mu_{1} \mu_{2} \alpha J_{1}+4 \alpha \mu_{1}^{2} J_{1}+\phi^{3} \mu_{1}+4 \mu_{1}^{2} \mu_{2} J_{1}+4 \theta \mu_{1}\left[\mu_{2}\left(\mathrm{~d}+\alpha+\mu_{1}\right)-\left(\beta_{2} S_{0} \mu_{2}+\beta_{1} S_{0} \eta\right)\right]\right.$ $+4 \alpha \theta \mu_{1} J_{1}+4 \mu_{1}^{2}\left[\mu_{2}\left(\mathrm{~d}+\alpha+\mu_{1}\right)-\left(\beta_{2} S_{0} \mu_{2}+\beta_{1} S_{0} \eta\right)\right]+2 \alpha \phi \mu_{1} J_{1}+\alpha \phi \mu_{2} J_{1}+\alpha \theta^{2} J_{1}+\theta^{2} \mu_{2} J_{1}+3 \phi^{2} \mu_{1} \mu_{2}$

$+\phi^{2} \mu_{1} J_{1}+9 \phi \mu_{1}^{2} \mu_{2}+4 \mu_{1}^{4}+10 \theta \phi \mu_{1}^{2}+\theta^{2}\left[\mu_{2}\left(\mathrm{~d}+\alpha+\mu_{1}\right)-\left(\beta_{2} S_{0} \mu_{2}+\beta_{1} S_{0} \eta\right)\right]+8 \phi \mu_{1}^{3}+6 \theta \phi \mu_{1} \mu_{2}$

$+5 \phi^{2} \mu_{1}^{2}+\phi^{2}\left[\mu_{2}\left(\mathrm{~d}+\alpha+\mu_{1}\right)-\left(\beta_{2} S_{0} \mu_{2}+\beta_{1} S_{0} \eta\right)\right]+9 \theta \mu_{1}^{2} \mu_{2}+4 \theta \mu_{1} \mu_{2} J_{1}+3 \theta \phi^{2} \mu_{1}+8 \theta \mu_{1}^{3}$

$+4 \phi \mu_{1}\left[\mu_{2} \cdot\left(\mathrm{d}+\alpha+\mu_{1}\right)-\left(\beta_{2} S_{0} \mu_{2}+\beta_{1} S_{0} \eta\right)\right]+5 \theta^{2} \mu_{1}^{2}+3 \phi \mu_{1} \cdot \mu_{2} J_{1}+6 \cdot \phi \mu_{1}^{2} J_{1}+3 \mathrm{~d} \mu_{1}^{2} J_{1}+2 \mathrm{~d} \phi \mu_{1} J_{1}$

$+2 \theta \phi\left[\mu_{2}\left(\mathrm{~d}+\alpha+\mu_{1}\right)-\left(\beta_{2} S_{0} \mu_{2}+\beta_{1} S_{0} \eta\right)\right]+8 \theta \mu_{1}^{2} J_{1}+3 \theta^{2} \phi \mu_{1}$

$+\theta \phi \mu_{2} J_{1}+\theta \phi \mu_{2} J_{1}+4 d \theta \mu_{1} J_{1}+\theta \phi \alpha J_{1}+6 \mu_{1}^{3} \mu_{2}+2 \theta^{2} \mu_{1} J_{1}+\mathrm{d} \mu_{1} \mu_{2} J_{1}+\theta^{3} \mu_{1}+3 \theta \phi \mu_{1} J_{1}+\mathrm{d} \phi \mu_{2} J_{1}+6 \mu_{1}^{3} J_{1}+\mathrm{d} \theta^{2} J_{1}$ $\left.+3 \theta^{2} \mu_{1} \mu_{2}\right\}+\left(\mathrm{d}+\alpha+\mu_{1}-J_{2}\right)\left\{4 \cdot \phi^{2} \mu_{1}^{3}+7 \mathrm{~d} \theta \phi \mu_{1}+\mathrm{d}^{2} \mu_{2} J_{1}^{2}+\theta \mathrm{d}^{2} J_{1}^{2}+3 \phi \mu_{1} \mu_{2} J_{1}^{2}+3 \phi \theta^{2} \mu_{1}^{2}+10 \phi^{2} \mu_{1}^{2} \mu_{2}+2 \alpha \theta \mathrm{d} J_{1}^{2}\right.$ $+2 \theta^{3} \mu_{1} \mu_{2}+\mathrm{d}^{2} \mu_{1} J_{1}^{2}+\mathrm{d} \phi \mu_{2} J_{1}^{2}+8 \theta \phi \mu_{1}^{3}+\theta \alpha^{2} J_{1}^{2}+8 \mu_{1}^{2} J_{1}\left[\mu_{2}\left(\mathrm{~d}+\alpha+\mu_{1}\right)-\left(\beta_{2} S_{0} \mu_{2}+\beta_{1} S_{0} \eta\right)\right]+2 \mathrm{~d} \alpha \mu_{1} J_{1}^{2}+9 \theta \mu_{1}^{2} \mu_{2}^{2}$ $+5 \mathrm{~d} \theta \mu_{1} \mu_{2} J_{1}+2 \mathrm{~d} \theta J_{1}\left[\mu_{2}\left(\mathrm{~d}+\alpha+\mu_{1}\right)-\left(\beta_{2} S_{0} \mu_{2}+\beta_{1} S_{0} \eta\right)\right]+6 \mu_{1}^{3} \mu_{2}^{2}+16 \phi \mu_{1}^{3} \mu_{2}+\alpha \theta^{2} \cdot J_{1}^{2}$ $+4 \mu_{1}^{3}\left[\mu_{2}\left(\mathrm{~d}+\alpha+\mu_{1}\right)-\left(\beta_{2} S_{0} \mu_{2}+\beta_{1} S_{0} \eta\right)\right]+6 \phi \theta^{2} \mu_{1} \mu_{2}+\theta^{2} \mu_{2} J_{1}^{2}+2 \mu_{1}^{5}+\theta^{2} \mu_{1} J_{1}^{2}+6 \mu_{1}^{3} J_{1}^{2}$ $+8 \cdot \phi \mu_{1} \mu_{2}\left[\mu_{2}\left(\mathrm{~d}+\alpha+\mu_{1}\right)-\left(\beta_{2} S_{0} \mu_{2}+\beta_{1} S_{0} \eta\right)\right]+4 \cdot \theta^{2} \mu_{1}\left[\mu_{2}\left(\mathrm{~d}+\alpha+\mu_{1}\right)-\left(\beta_{2} S_{0} \mu_{2}+\beta_{1} S_{0} \eta\right)\right]$ $+\phi\left[\mu_{2}\left(\mathrm{~d}+\alpha+\mu_{1}\right)-\left(\beta_{2} S_{0} \mu_{2}+\beta_{1} S_{0} \eta\right)\right]^{2}+2 \alpha \phi \mu_{2}^{2} J_{1}+\phi^{2} \cdot J_{1} \cdot\left[\mu_{2}\left(\mathrm{~d}+\alpha+\mu_{1}\right)-\left(\beta_{2} S_{0} \mu_{2}+\beta_{1} S_{0} \eta\right)\right]$ $+5 \theta \alpha \mu_{1} \cdot \mu_{2} J_{1}+5 \mathrm{~d} \mu_{1}^{3} J_{1}+2 \alpha \phi \mu_{1} J_{1}^{2}+2 \mathrm{~d} \phi \mu_{1} J_{1}^{2}+2 \mu_{1} \mu_{2}^{2} J_{1}^{2}+8 \mu_{1}^{2} \mu_{2}^{2} J_{1}+\theta \phi \mu_{2} J_{1}^{2}$ $+4 \phi^{2} \mu_{1}\left[\mu_{2}\left(\mathrm{~d}+\alpha+\mu_{1}\right)-\left(\beta_{2} S_{0} \mu_{2}+\beta_{1} S_{0} \eta\right)\right]+\alpha \phi^{2} \mu_{2} J_{1}+\theta \phi^{2} \mu_{1} J_{1}+14 \theta \mu_{1}^{3} \cdot J_{1}+8 \mu_{1}^{2} \mu_{2} J_{1}^{2}+5 \alpha \theta^{2} \mu_{1} J_{1}$ $+2 \theta \mu_{2} J_{1}\left[\mu_{2}\left(\mathrm{~d}+\alpha+\mu_{1}\right)-\left(\beta_{2} S_{0} \mu_{2}+\beta_{1} S_{0} \eta\right)\right]+\theta \alpha \phi J_{1}^{2}+20 \theta \phi \mu_{1}^{2} \mu_{2}$ $+\theta^{2} J_{1}\left[\mu_{2}\left(\mathrm{~d}+\alpha+\mu_{1}\right)-\left(\beta_{2} S_{0} \mu_{2}+\beta_{1} S_{0} \eta\right)\right]+6 \cdot \alpha \phi \mu_{1}^{2} J_{1}+8 \theta \mu_{1} \mu_{2}\left[\mu_{2}\left(\mathrm{~d}+\alpha+\mu_{1}\right)-\left(\beta_{2} S_{0} \mu_{2}+\beta_{1} \cdot S_{0} \eta\right)\right]$ $+2 \alpha \phi^{2} \mu_{1} J_{1}+\mathrm{d} \phi^{2} \mu_{2} J_{1}+\mathrm{d} \theta \phi J_{1}^{2}+\alpha \mu_{2}^{2} J_{1}^{2}+3 \theta^{2} \mu_{1} \mu_{2}^{2}+7 \alpha \theta \mu_{1} J_{1}^{2}+\mathrm{d} \theta \phi^{2} J_{1}+9 \phi \mu_{1}^{2} \mu_{2}^{2}+6 \theta \mu_{1} \cdot \mu_{2} J_{1}^{2}+16 \theta \mu_{1}^{3} \mu_{2}$ $+6 \mathrm{~d} \mu_{1}^{2} J_{1}^{2}+5 \phi \mu_{1}^{4}+9 \mathrm{~d} \theta \mu_{1}^{2} J_{1}+d \theta^{2} J_{1}^{2}+5 \alpha \phi \mu_{1} \mu_{2} J_{1}+2 \theta \phi J_{1}\left[\mu_{2}\left(\mathrm{~d}+\alpha+\mu_{1}\right)-\left(\beta_{2} \cdot S_{0} \mu_{2}+\beta_{1} S_{0} \eta\right)\right]$ $+5 \phi \mu_{1} J_{1}\left[\mu_{2}\left(\mathrm{~d}+\alpha+\mu_{1}\right)-\left(\beta_{2} S_{0} \mu_{2}+\beta_{1} S_{0} \eta\right)\right]+5 \theta \mu_{1}^{4}+4 \phi^{2} \mu_{1}^{2} J_{1}+\mathrm{d} \theta^{3} J_{1}+2 \phi^{3} \mu_{1} \mu_{2}+7 \alpha \theta \phi \mu_{1} J_{1}+3 \phi^{2} \mu_{1} \mu_{2}^{2}$ $+5 \mathrm{~d} \phi \mu_{1} \mu_{2}+13 \theta \phi \mu_{1} \mu_{2} J_{1}+\theta^{3} \mu_{2} J_{1}+\theta^{3} \mu_{1} J_{1}+2 \mathrm{~d} \theta \phi \mu_{2} J_{1}+\alpha \phi \mu_{2} J_{1}^{2}+\alpha \phi J_{1}\left[\mu_{2} \cdot\left(\mathrm{d}+\alpha+\mu_{1}\right)-\left(\beta_{2} S_{0} \mu_{2}+\beta_{1} S_{0} \eta\right)\right]$ $+6 \theta \mu_{1}^{2}\left[\mu_{2}\left(\mathrm{~d}+\alpha+\mu_{1}\right)-\left(\beta_{2} S_{0} \mu_{2}+\beta_{1} S_{0} \eta\right)\right]+\theta \phi \mu_{1} J_{1}^{2}+5 \alpha \mu_{1} \mu_{2} J_{1}^{2}+2 \phi^{2} \mu_{2}\left[\mu_{2}\left(\mathrm{~d}+\alpha+\mu_{1}\right)-\left(\beta_{2} S_{0} \mu_{2}+\beta_{1} S_{0} \eta\right)\right]$ $+\phi^{3}\left[\mu_{2} \cdot\left(\mathrm{d}+\alpha+\mu_{1}\right)-\left(\beta_{2} S_{0} \mu_{2}+\beta_{1} S_{0} \eta\right)\right]+\phi \mu_{2}\left[\mu_{2}\left(\mathrm{~d}+\alpha+\mu_{1}\right)-\left(\beta_{2} S_{0} \mu_{2}+\beta_{1} S_{0} \eta\right)\right]+22 \theta \mu_{1}^{2} \mu_{2} J_{1}+5 \phi^{2} \mu_{1} \mu_{2} J_{1}$ $+2 \theta^{2} \mu_{2}\left[\mu_{2}\left(\mathrm{~d}+\alpha+\mu_{1}\right)-\left(\beta_{2} S_{0} \mu_{2}+\beta_{1} S_{0} \eta\right)\right]+5 \alpha \cdot \mu_{1}^{2} \mu_{2} J_{1}+8 \theta^{2} \mu_{1}^{2} \mu_{2} J_{1}+8 \theta^{2} \mu_{1}^{2} J_{1}+5 \mathrm{~d} \theta^{2} \mu_{1} J_{1}$ $+6 \cdot \phi \mu_{1}^{2}\left[\mu_{2}\left(\mathrm{~d}+\alpha+\mu_{1}\right)-\left(\beta_{2} S_{0} \mu_{2}+\beta_{1} S_{0} \eta\right)\right]+\alpha \theta \phi^{2} J_{1}+2 \alpha \theta \phi \mu_{2} J_{1}+\mathrm{d} \theta \mu_{2} J_{1}^{2}+9 \alpha \theta \mu_{1}^{2} J_{1}+\alpha \theta \mu_{2} J_{1}^{2}$ $+2 \mathrm{~d} \cdot \mu_{1} \mu_{2}^{2} J_{1}+8 \mu_{1}^{4} J_{1}+6 \alpha \mu_{1}^{2} J_{1}^{2}+19 \phi \mu_{1}^{2} \mu_{2} J_{1}+\alpha \mu_{2} J_{1} \cdot\left[\mu_{2}\left(\mathrm{~d}+\alpha+\mu_{1}\right)-\left(\beta_{2} S_{0} \mu_{2}+\beta_{1} S_{0} \eta\right)\right]$ $+6 \mathrm{~d} \phi \mu_{1}^{2} J_{1}+8 \theta \mu_{1} \mu_{2}^{2} J_{1}+2 \mathrm{~d} \theta^{2} \phi J_{1}+4 \theta \phi \mu_{2}\left[\mu_{2}\left(\mathrm{~d}+\alpha+\mu_{1}\right)-\left(\beta_{2} S_{0} \mu_{2}+\beta_{1} S_{0} \eta\right)\right]$ $+6 \theta \mu_{1} J_{1}\left[\mu_{2}\left(\mathrm{~d}+\alpha+\mu_{1}\right)-\left(\beta_{2} S_{0} \cdot \mu_{2}+\beta_{1} S_{0} \eta\right)\right]+4 \theta^{2} \mu_{2}^{3}+5 \mathrm{~d} \mu_{1} \mu_{2} J_{1}^{2}$ $+4 \mu_{1} \mu_{2} J_{1}\left[\mu_{2}\left(\mathrm{~d}+\alpha+\mu_{1}\right)-\left(\beta_{2} S_{0} \mu_{2}+\beta_{1} S_{0} \eta\right)\right]+6 \theta \mu_{1} J_{1}\left[\mu_{2}\left(\mathrm{~d}+\alpha+\mu_{1}\right)-\left(\beta_{2} S_{0} \mu_{2}+\beta_{1} S_{0} \eta\right)\right]$ $+4 \theta^{2} \mu_{2}^{2}+5 \mathrm{~d} \mu_{1} \mu_{2} J_{1}^{2}+4 \cdot \mu_{1} \mu_{2} J_{1}\left[\mu_{2}\left(\mathrm{~d}+\alpha+\mu_{1}\right)-\left(\beta_{2} S_{0} \mu_{2}+\beta_{1} S_{0} \eta\right)\right]$ $+3 \mathrm{~d} \mu_{1} \cdot J_{1}\left[\mu_{2}\left(\mathrm{~d}+\alpha+\mu_{1}\right)-\left(\beta_{2} S_{0} \mu_{2}+\beta_{1} S_{0} \eta\right)\right]+2 \alpha \mu_{1} \mu_{2}^{2} J_{1}+7 \theta \mu_{1}^{2} J_{1}^{2}+\alpha^{2} \mu_{2} J_{1}^{2}+\alpha \theta^{3} J_{1}+\phi^{3} \mu_{1}^{2}+2 \theta^{2} \phi \mu_{1} J_{1}$ $+3 \phi \mu_{1}^{2} J_{1}^{2}+3 \theta \phi^{2} \mu_{1}^{2}+\theta^{3} \mu_{1}^{2}+2 \alpha \theta J_{1}\left[\mu_{2}\left(\mathrm{~d}+\alpha+\mu_{1}\right)-\left(\beta_{2} S_{0} \mu_{2}+\beta_{1} S_{0} \eta\right)\right]+\theta \phi^{2} \mu_{2} J_{1}+8 \mu_{1}^{4} \mu_{2}+16 \mu_{1}^{3} \mu_{2} \cdot J_{1}+7 \theta^{2} \mu_{1}^{2} J_{1}$ 


$$
\begin{aligned}
& +d \phi J_{1}\left[\mu_{2}\left(\mathrm{~d}+\alpha+\mu_{1}\right)-\left(\beta_{2} S_{0} \mu_{2}+\beta_{1} S_{0} \eta\right)\right]+7 \mathrm{~d} \theta \mu_{1} J_{1}^{2}+2 \alpha \phi \theta^{2} J_{1}+\mathrm{d} \mu_{2} J_{1}\left[\mu_{2}\left(\mathrm{~d}+\alpha+\mu_{1}\right)-\left(\beta_{2} S_{0} \mu_{2}+\beta_{1} S_{0} \eta\right)\right] \\
& +3 \alpha \mu_{1} J_{1}\left[\mu_{2}\left(\mathrm{~d}+\alpha+\mu_{1}\right)-\left(\beta_{2} S_{0} \mu_{2}+\beta_{1} S_{0} \eta\right)\right]+\alpha \theta^{2} \mu_{2} J_{1}+5 \mathrm{~d} \mu_{1}^{2} \mu_{2} J_{1}+5 \cdot \alpha \mu_{1}^{3} J_{1}+6 \phi \mu_{1} \mu_{2}^{3} J_{1} \\
& +2 \mu_{1}\left[\mu_{2}\left(\mathrm{~d}+\alpha+\mu_{1}\right)-\left(\beta_{2} \cdot S_{0} \mu_{2}+\beta_{1} S_{0} \eta\right)\right]+2 \mathrm{~d} \phi^{2} \mu_{1} J_{1}+11 \theta \phi \mu_{1}^{2} J_{1}+\alpha^{2} \mu_{1} J_{1}^{2}+2 \theta^{2} \mu_{2}^{2} J_{1}+\mathrm{d} \theta^{2} \mu_{2} J_{1} \\
& +3 \theta \phi^{3}\left[\mu_{2}\left(\mathrm{~d}+\alpha+\mu_{1}\right)-\left(\beta_{2} S_{0} \mu_{2}+\beta_{1} S_{0} \eta\right)\right]+10 \theta^{2} \mu_{1}^{2} \mu_{2}+3 \theta^{2} \phi\left[\mu_{2}\left(\mathrm{~d}+\alpha+\mu_{1}\right)-\left(\beta_{2} S_{0} \mu_{2}+\beta_{1} S_{0} \eta\right)\right] \\
& +8 \theta \phi \mu_{1}\left[\mu_{2}\left(\mathrm{~d}+\alpha+\mu_{1}\right)-\left(\beta_{2} S_{0} \mu_{2}+\beta_{1} S_{0} \eta\right)\right]+11 \phi \mu_{1}^{3} J_{1}+\theta \mu_{2}^{2} J_{1}^{2}+\mathrm{d} \mu_{2}^{2} \cdot J_{1}^{2}+6 \theta \phi \mu_{1} \mu_{2}^{2} \\
& +\theta^{3}\left[\mu_{2}\left(\mathrm{~d}+\alpha+\mu_{1}\right)-\left(\beta_{2} S_{0} \mu_{2}+\beta_{1} S_{0} \eta\right)\right]+2 \theta \phi \mu_{2}^{2} J_{1}+2 \mathrm{~d} \alpha \mu_{2} J_{1}^{2}+2 \theta^{2} \phi \mu_{2} J_{1} \\
& \left.+\theta \cdot\left[\mu_{2}\left(\mathrm{~d}+\alpha+\mu_{1}\right)-\left(\beta_{2} S_{0} \mu_{2}+\beta_{1} S_{0} \eta\right)\right]+8 \mu_{1}^{2} \mu_{2}\left[\mu_{2} \cdot\left(\mathrm{d}+\alpha+\mu_{1}\right)-\left(\beta_{2} S_{0} \mu_{2}+\beta_{1} S_{0} \eta\right)\right]\right\} \\
& +\left[\mu_{2}\left(\mathrm{~d}+\alpha+\mu_{1}\right)-\left(\beta_{2} S_{0} \mu_{2}+\beta_{1} S_{0} \eta\right)\right]^{2}\left\{\phi \mu_{2}+2 \mu_{1} \mu_{2}+\theta J_{1}+\phi J_{1}+\theta \mu_{2}+2 \mu_{1} J_{1}\right\} \\
& +\left[\mu_{2}\left(\mathrm{~d}+\alpha+\mu_{1}\right)-\left(\beta_{2} S_{0} \mu_{2}+\beta_{1} \cdot S_{0} \eta\right)\right]\left\{8 \theta \phi \mu_{1} \mu_{2}+3 \theta \phi \mu_{2} J_{1}+3 \mathrm{~d} \mu_{1} J_{1}^{2}+4 \phi \mu_{1} \mu_{2}^{2}+2 \theta \mu_{1}^{2} J_{1}+\phi^{2} \mu_{2}^{2}\right. \\
& +4 \theta^{2} \mu_{1} \mu_{2}+\alpha \theta \phi J_{1}+\alpha \theta \mu_{1} J_{1}+\alpha \cdot \mu_{2}^{2} J_{1}+4 \mu_{1}^{3} \mu_{2}+8 \mu_{1}^{2} \mu_{2} J_{1}+\phi \mu_{2} J_{1}^{2}+6 \theta \mu_{1}^{2} \mu_{2}+\alpha \mu_{2} J_{1}^{2}+3 \alpha \phi \mu_{1} J_{1} \\
& +6 \theta \mu_{1} \mu_{2} J_{1}+\mathrm{d} \mu_{2} J_{1}^{2}+\theta^{2} \mu_{2} J_{1}+4 \mu_{1}^{2} \mu_{2}^{2}+\mathrm{d} \mu_{2}^{2} J_{1}+4 \mu_{1}^{2} J_{1}^{2}+\mathrm{d} \theta \phi J_{1}+\phi^{3} \mu_{2}+\alpha \cdot \phi^{2} J_{1}+3 \theta \phi^{2} \mu_{2}+2 \alpha \theta J_{1}^{2} \\
& +2 \theta \mu_{2} J_{1}^{2}+\phi^{2} \mu_{1} J_{1}+\alpha \mu_{1} \cdot \mu_{2} J_{1}+\phi \mu_{1} J_{1}^{2}+2 \theta \phi \mu_{2}^{2}+\mathrm{d} \phi J_{1}^{2}+4 \phi^{2} \mu_{1} \mu_{2}+4 \theta \mu_{1} \cdot \mu_{2}^{2}+\mathrm{d} \theta \mu_{1} J_{1}+\mathrm{d} \mu_{1} \mu_{2} J_{1}+\theta^{3} \mu_{2} \\
& +\alpha \phi J_{1}^{2}+\phi \mu_{2}^{2} J_{1}+4 \mu_{1} \mu_{2}^{2} J_{1}+4 \mu_{1} \mu_{2} J_{1}^{2}+2 \alpha \mu_{1}^{2} J_{1}+6 \phi \mu_{1}^{2} \mu_{2}+3 \theta^{2} \phi \mu_{2}+4 \phi \mu_{1}^{2} J_{1}+3 \alpha \mu_{1} J_{1}^{2}+\mathrm{d} \phi^{2} J_{1}+2 \mathrm{~d} \theta J_{1}^{2} \\
& +2 \mathrm{~d} \mu_{1}^{3} J_{1}^{2}+2 \phi \theta^{2} \mu_{2}^{2} J_{1}+13 \phi \mu_{1}^{3} \mu_{2} J_{1}+\theta \mu_{2}^{2} J_{1}^{3}+5 \alpha \theta \phi \mu_{1}^{2} J_{1}+3 \cdot \mathrm{d} \phi \mu_{1}^{3} J_{1}+2 \theta^{2} \phi \mu_{1} \mu_{2} J_{1}+4 \alpha \theta^{2} \mu_{1}^{2} J_{1}+5 \theta \phi \mu_{1}^{3} J_{1} \\
& +10 \cdot \theta \phi \mu_{1} \mu_{2}^{2} J_{1}+3 \alpha \mu_{1}^{3} \mu_{2} J_{1}+4 \phi^{2} \mu_{1} \mu_{2}^{2} J_{1}+\alpha \theta \mu_{1} \mu_{2}^{2} J_{1}+2 \theta \phi \mu_{2}^{2} J_{1}^{2}+2 \mathrm{~d} \alpha \theta \phi J_{1}^{2}+\alpha \theta \phi^{2} \mu_{1} J_{1}+\theta^{3} \mu_{2}^{2} J_{1} \\
& +4 \cdot \alpha \theta \phi \mu_{1} \mu_{2}^{2} J_{1}+\theta \phi \mu_{2}^{3} J_{1}+3 \mathrm{~d} \theta \phi \mu_{1} \mu_{2} J_{1}+12 \theta \phi \mu_{1}^{2} \mu_{2} J_{1}+14 \theta \mu_{1}^{2} \mu_{2}^{2} J_{1}^{2}+\theta^{3} \mu_{1} \mu_{2} J_{1}+\alpha \theta^{3} \mu_{1} J_{1}+\mathrm{d} \theta^{3} \mu_{1} J_{1} \\
& +\theta^{3} \cdot \mu_{2}^{2} J_{1}+2 \mathrm{~d} \alpha \mu_{2}^{2} J_{1}+2 \mathrm{~d} \alpha \mu_{1} \mu_{2} J_{1}^{2}+\mathrm{d} \alpha \mu_{1} \mu_{2}^{3} J_{1}+3 \mathrm{~d} \phi \mu_{1} \mu_{2}^{3} J_{1}+\theta \phi^{2} \mu_{1} \mu_{2} J_{1}+3 \alpha \phi \mu_{1}^{3} J_{1}+\mathrm{d} \phi \mu_{2}^{3} J_{1} \\
& +2 \mathrm{~d} \mu_{1}^{2} \cdot \mu_{2}^{2} J_{1}+3 \mathrm{~d} \theta \mu_{1} J_{1}^{3}+5 \mathrm{~d} \phi \mu_{1}^{2} \mu_{2} J_{1}+\mathrm{d} \theta \phi \mu_{2}^{2} J_{1}+8 \mu_{1}^{2} \cdot \mu_{2}^{2} J_{1}^{2}+5 \alpha \theta \mu_{1}^{3} J_{1}+7 \theta^{2} \mu_{1} \mu_{2} J_{1}+\theta^{3} \mu_{1} \mu_{2}^{2} \\
& +4 \mu_{1}^{2} \cdot \mu_{2} J_{1}^{2} 4 \theta \mu_{1} \mu_{2}^{3} J_{1}+5 \alpha \mu_{1}^{3} J_{1}^{2}+\mathrm{d}^{2} \phi \mu_{2} J_{1}^{2}+3 \mathrm{~d} \mu_{1} \mu_{2}^{2} \cdot J_{1}^{2}+3 \phi \mu_{1} \cdot \mu_{2}^{3} J_{1}+3 \alpha \theta \phi \mu_{1} \mu_{2} J_{1}+4 \mu_{1}^{4} J_{1}^{2} \\
& +\theta^{3} \mu_{1}^{2} \cdot \mu_{2}+\theta \mu_{2}^{2} J_{1}+\mathrm{d} \cdot \theta \mu_{2} J_{1}+3 \mathrm{~d} \phi \mu_{1} J_{1}+2 \phi^{2} \mu_{2} J_{1}+\alpha \theta \cdot \mu_{2} J_{1}+2 \mathrm{~d} \mu_{1}^{2} J_{1}+2 \theta \mu_{1} \cdot J_{1}^{2}+4 \mu_{1}^{3} J_{1} \\
& \left.+\theta \phi \mu_{1} J_{1}+7 \phi \cdot \mu_{1} \mu_{2} J_{1}\right\}+(1-\mathrm{d}) \theta^{2} J_{1}^{2} \mu_{2}+\left(\mu_{2}^{2}-\alpha \theta^{2}\right) \alpha J_{1}^{2} \mu_{2}+2 \mathrm{~d} \cdot \theta^{2} J_{1}^{2} \mu_{1}+2 \alpha \theta^{2} J_{1}^{2} \mu_{1}+\alpha \theta \phi \mu_{2}^{2} J_{1} \\
& +\phi \alpha^{2} J_{1}^{2} \mu_{1}+3 \mathrm{~d} \cdot \mu_{1}^{3} \mu_{2} J_{1}+\phi^{2} \mu_{1}^{3} J_{1}+8 \phi \mu_{1}^{2} \mu_{2} J_{1}^{2}+\theta^{3} \mu_{1}^{2} J_{1}+3 \mathrm{~d} \mu_{1}^{2} \cdot J_{1}^{3}+\alpha \theta \mu_{2} J_{1}^{2}+\mathrm{d} \theta \mu_{2} J_{1}^{3}+5 \mathrm{~d} \theta \phi \mu_{1}^{2} J_{1} \\
& +\alpha^{2} \theta \phi J_{1}^{2}+\phi \mu_{1} \mu_{2}^{2} J_{1}^{2}+2 \mathrm{~d} \phi \mu_{2}^{2} J_{1}^{2}+2 \mathrm{~d} \alpha \theta \mu_{1} J_{1}^{2}+3 \mathrm{~d} \theta \mu_{1} \mu_{2} J_{1}^{2}+4 \mathrm{~d} \phi \mu_{1}^{2} J_{1}^{2}+\mathrm{d}^{2} \phi \mu_{1} J_{1}^{2}+2 \theta \phi \mu_{1} \mu_{2}^{3} \\
& +6 \theta \mu_{1}^{3} J_{1}^{2}+8 \cdot \mu_{1}^{3} \mu_{2}^{2}(\theta+\phi)+2 \mathrm{~d} \phi^{2} \mu_{1} \mu_{2} J_{1}+5 \mu_{1}^{2}\left(\theta^{2}+\phi^{2}\right)+2 \theta^{2} \cdot \mu_{1}^{2} J_{1}^{2}+4 \phi^{2} \mu_{1}^{3} \mu_{2}+2 \mu_{1}^{5} \mu_{2}+3 \phi \mu_{1}^{3} J_{1}^{2} \\
& +4 \alpha \phi \mu_{1}^{2} J_{1}^{2}+13 \phi \mu_{1}^{2} \mu_{2}^{2} J_{1}+\alpha \phi^{2} \mu_{1}^{2} J_{1}+\mathrm{d}^{2} \theta \phi J_{1}^{2}+\theta \alpha^{2} \mu_{1} J_{1}^{2}+3 \mu_{1}^{2} \mu_{2}^{3}(\theta+\phi)+7 \mathrm{~d} \mu_{1}^{2} \mu_{2} J_{1}^{2}+4 \mathrm{~d} \theta^{2} \mu_{1}^{2} J_{1} \\
& +\mathrm{d} \theta \mu_{1} \mu_{2}^{2} J_{1}+\mathrm{d}^{2} \mu_{1}^{2} J_{1}^{2}+\phi^{2} \mu_{1} \mu_{2}^{3}+2 \alpha \mu_{1}^{2} \mu_{2}^{2} J_{1}+6 \theta^{2} \mu_{1} \mu_{2}^{2} J_{1}+2 \mathrm{~d} \alpha \phi \mu_{1} J_{1}^{2} .
\end{aligned}
$$

From Section 5, we know that

$$
\mu_{2}\left(\mathrm{~d}+\alpha+\mu_{1}\right)=\mu_{2} J_{2}+\eta J_{3}
$$

and

$$
\mathrm{d}+\alpha+\mu_{1}>J_{2} .
$$

After some algebraic manipulations, we have $b_{1} b_{2} b_{3}-b_{3}^{2}-b_{1}^{2} b_{4}>0$, Thus, $b_{1} b_{2} b_{3}>b_{3}^{2}+b_{1}^{2} b_{4}$, when $R_{v}>1$. 